\title{
ANÁLISE TÉRMICA DE UM ECO COMPÓSITO DE POLIURETANO DE MAMONA COM REJEITO DE MADEIRA
}

\author{
F. M. BARRETO*, R. A. D. CUNHA E J. U. L. MENDES \\ Universidade Federal do Rio Grande do Norte \\ fernanda_mbarreto@hotmail.com*
}

Artigo submetido em dezembro/2015 e aceito em setembro/2016

DOI: $10.15628 /$ holos. 2016.3840

\section{RESUMO}

Este trabalho tem como objetivo realizar uma análise comparativa entre materiais compósitos, cuja finalidade é para isolamento térmico, os mesmos foram confeccionados com matriz polimérica de poliuretano (PU) a base de mamona e como material de reforço utilizou madeira, esta em duas granulometrias distintas, na forma de pó e na forma de raspa, ambas as configurações do compósito, foram usados $20 \%$ do reforço. Após confecção dos corpos de provas, foram realizados o ensaio de densidade aparente e os ensaios térmicos. Através dos ensaios térmicos, tornou-se possível uma avaliação no comportamento de propriedades térmicas, como calor específico, difusividade térmica, condutividade térmica e resistividade térmica. Com o estudo, concluímos que os materiais reforçados com madeira apresentam boas características térmicas, podendo estes serem aplicados como isolantes térmicos.

PALAVRAS-CHAVE: Eco-compósito, Poliuretano de Mamona, Rejeito de Madeira, Propriedades Térmicas.

\section{THERMAL ANALYSIS OF AN ECOLOGICAL COMPOSITE OF CASTOR BEANS'S POLYURETHANE WITH WOOD WASTE}

\begin{abstract}
This paper aims to conduct a comparative analysis of composite materials subjected to apparently density tests and thermal tests. It will be made three samples. These materials will have as polymer matrix polyurethane (PU) with base of castor beans. A sample will be done with pure PU and the other two will be reinforced with sawdust and other wood-shaving in the proportion of $20 \%$ of the body made of pure PU. The PU was obtained
\end{abstract}

commercially, while the wood was recycled from the tailings joinery. Through the thermal tests, it became possible to evaluate the behavior of its thermal properties. The fabrications of composites were made in metallic molds. With the study, we concluded that the materials reinforced with wood have a lower thermal conductivity and they are more apt to use as thermal insulation.

KEYWORDS: Composite, Polyurethane, Wood, Density, Thermal Properties. 


\section{INTRODUÇÃO}

Os compósitos surgiram da necessidade de se obter materiais que combinem as propriedades dos materiais existentes. Deste modo, de acordo com as características pretendidas, os compósitos são desenvolvidos em função de cada aplicação particular.

O compósito estudado neste trabalho tem como finalidade isolação térmica. Segundo INCROPERA (2008), os isolantes térmicos são constituídos por materiais de baixa condutividade térmica, os combinado para se obter um sistema com uma condutividade térmica ainda menor. Um importante parâmetro do sistema é a sua densidade aparente (massa do sólido/volume total), que depende fortemente da forma na qual o material está empacotado. Em todos os tipos de isolantes, vácuo nos espaços vazios implica na redução da condutividade térmica efetiva do sistema.

A eficiência isolante de um material varia na razão inversa do seu peso específico. Como o frio é difícil de produzir, há necessidade de isolar bem as paredes de câmaras frias, onde devemse empregar materiais de baixa densidade, condutividade térmica baixa, não higroscópicos, imputrescíveis, com grande poder de reflexão, estáveis entre certos limites de temperatura, não inflamáveis e inertes em relação à madeira ou ao ferro, quando em contato com os mesmos (RAPIN, 2001).

Diante disso, se faz necessário o estudo das propriedades térmicas dos materiais, como: condutividade, calor específico, difusividade e resistividade para entender qual material funciona melhor como isolante térmico.

O seguinte trabalho procura desenvolver um novo tipo de material no mercado. Material esse, que reutiliza substâncias que seriam rejeitadas, fazendo uma espécie de reciclagem, além da fabricação de um material em partes ecológico, para uma futura utilização. Uma análise comparativa nas propriedades térmicas será feita a partir dados coletados dos corpos de prova, os quais foram submetidos a ensaios térmicos de condutividade, calor específico, difusividade e resistividade.

\section{REVISÃO BIBLIOGRÁFICA}

Nesse capítulo iremos falar um pouco sobre os materiais compósitos, sua definição e classificação, sobre a definição de algumas propriedades térmicas e referenciar estudos de alguns autores nessa área.

\subsection{Compósitos}

Algumas definições, classificações e alguns conceitos básicos, desse tipo de material, são necessários para o entendimento deste trabalho. Também será tratado como surgiu e como se deu a evolução dos materiais compósitos nos setores industriais e o quanto esses materiais estão presentes no nosso dia a dia.

A madeira, o bambu e até o próprio corpo humano são exemplos de materiais compostos (HERAKOVICH, 1997). Dessa forma, os compósitos podem ser considerados tão ou mais antigos do 
que o homem. Esses citados podem ser classificados como naturais, ou seja, feitos pela própria natureza.

A preparação de compósitos de madeira com polímeros é uma prática antiga, particularmente quanto ao uso de resinas termofixas na produção de painéis MDF (mediumdensity fiberboard). A utilização de farinha ou fibra de madeira como carga em termoplásticos, também já é conhecida desde a década de 70 pela indústria automobilística, que emprega compósitos de polipropileno com farinha de madeira, conhecidos no mercado, como Woodstock (CORREA, 2003).

Segundo FREIRE JR. (2005), os compósitos poliméricos, também conhecidos como plásticos reforçados, são os mais proeminentes dentre os materiais compósitos. Foram introduzidos por volta da década de $40 \mathrm{com}$ a necessidade do desenvolvimento de estruturas específicas durante a segunda guerra mundial. Já no final da década de 40, os compósitos de resina fenólica reforçada com fibra de vidro ganharam destaque, e, deram início ao uso desses materiais no projeto de estruturas.

\subsubsection{Definição}

Em geral, podemos definir como material compósito a junção de dois ou mais materiais que possuam fases distintas, ou seja, heterogêneos. Esse tem finalidade de obter propriedades específicas e características desejadas devido a essa interação. Em seguida, temos algumas definições de materiais compósitos, de acordo com algumas referências:

1) Para SHACKELFORD (2008), esses materiais envolvem alguma combinação de dois ou mais componentes dos tipos fundamentais de material (metais, vidro, cerâmicas e polímeros). Uma filosofia chave na seleção de materiais compósitos é de que eles devem fornecer o 'melhor dos dois mundos', ou seja, as propriedades atraentes de cada componente.

2) De acordo com CALLYSTER (2002), de uma maneira geral, pode-se considerar um compósito como sendo qualquer material multifásico que exiba uma proporção significativa das propriedades de ambas as fases que o constituem, de tal modo que é obtida uma melhor combinação de propriedades. Muitos materiais compósitos são constituídos por apenas duas fases: uma chamada matriz, que é continua e envolve a outra fase denominada reforço.

3) MENDONÇA (2005) diz que material compósito é um sistema combinado por dois ou mais materiais a nível macroscópico que trabalham simultaneamente, funcionado como uma unidade visando obter um conjunto de propriedades que nenhum dos componentes individualmente apresentaria, sendo formado por um reforço caracterizado objetivando influenciar na resistência mecânica do material, e a matriz que é responsável por transmitir os esforços ao reforço e dá a forma ao elemento estrutural.

Com esses conceitos, verifica-se que um compósito tem como objetivo a fabricação de um material com excelentes propriedades físicas e mecânicas, as quais juntas não podem ser encontradas em um material fundamental. Essas propriedades podem se adequar a projetos a partir da variação das proporções dos elementos utilizados durante sua fabricação. 


\subsection{Isolantes Térmicos}

Segundo TORREIRA (1980), a função primária de um isolante térmico é reduzir a taxa de transferência de calor entre um sistema e o meio, de modo que a energia possa ser conservada. Para isto, partes dos sistemas térmicos devem ser revestidas com material que possua propriedades e espessura, tais que a temperatura se mantenha dentro de um determinado intervalo.

Atualmente existe enorme quantidade de materiais classificados como isolantes térmicos, eles podem se classificar em: naturais, sintéticos e minerais.

\subsection{Propriedades Térmicas}

As três propriedades físicas de um material mais importante de um ponto de vista de cálculos térmicos são: a condutividade térmica, a difusividade térmica e o calor específico. Estas três propriedades estão relacionadas entre si pela Eq. 1 (GALVÃO,2014):

$\propto=\frac{k}{\rho c}$

Onde:

$\propto$ é a difusividade térmica $\left(\mathrm{m}^{2} / \mathrm{s}\right)$;

$k$ é a condutividade térmica $(\mathrm{W} / \mathrm{m}-\mathrm{K})$;

$\rho$ é a massa específica $\left(\mathrm{kg} / \mathrm{m}^{3}\right)$;

$c$ é o calor específico $(\mathrm{J} / \mathrm{kg}-\mathrm{K})$.

\subsubsection{Condutividade Térmica}

A condução é um processo de transferência de energia, ao longo do sistema, partícula a partícula. Uma partícula ao receber energia aumenta seu estado de vibração e dessa forma, acaba por transferir energia às partículas vizinhas. O coeficiente de condutividade térmica $(k)$ é característica de cada material. Os bons condutores têm $\mathrm{k}$ elevado, enquanto que os maus condutores, reduzido (GALVÃO, 2014).

\subsubsection{Calor Específico}

O calor específico é uma grandeza escalar que mede a dificuldade da substância variar sua energia, também podendo ser definida como a capacidade térmica por unidade de massa. É também uma propriedade crítica em muitas aplicações (GALVÃO, 2014).

\subsubsection{Difusividade Térmica}

A difusividade térmica é uma medida da rapidez com qual o calor se propaga no material (GALVÃO, 2014).

Ou seja, ela mede a capacidade do material de conduzir energia térmica em relação à sua capacidade de armazená-la (INCROPERA, 2008). 


\section{METODOLOGIA}

\subsection{Matriz Polimérica}

O polímero utilizado como matriz foi o poliuretano semiflexível, o mesmo foi adquirido comercialmente, como nome comercial de RESPAN SFD. Sua composição possui ésteres do ácido ricinoleico e prepolímeros derivados do óleo de mamona (dados do fabricante). Para a obtenção desse polímero se fez necessário à mistura de dois compostos poliméricos chamados de componentes A e B (Fig. 1), o componente B possui uma coloração mais escura. Estes componentes se combinam na proporção 1:0,5 (A:B) para reagirem e formarem a espuma semiflexível.

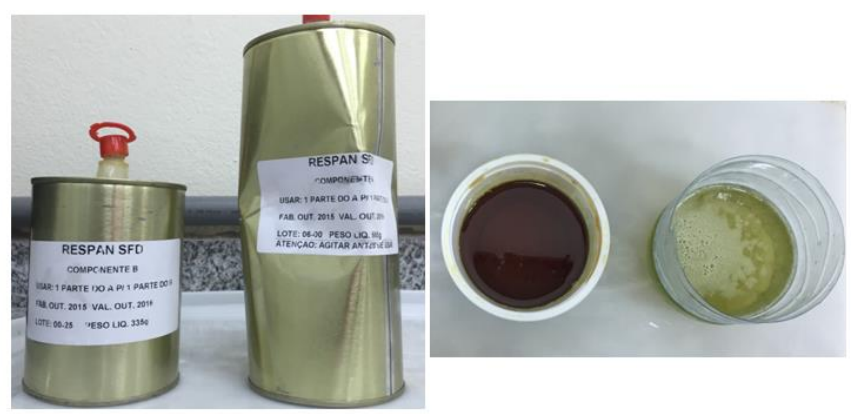

Figura 1: Componentes A e B do poliuretano.

\subsection{Material de Reforço}

Com uma visão de sustentabilidade e pensando no meio ambiente, a utilização da madeira que foi empregada como reforço na forma particulada em diferentes configurações e granulometria (Fig. 2), podem ser encontradas em marcenarias comuns, que descartam as mesmas e em algumas ocasiões vendem a empresas de fabricação de tijolos e telhas servindo como combustível fóssil. Deve salientar que as duas formas de madeira usadas no trabalho foram na forma de pó e raspa. E que a porcentagem usada nos corpos de prova foi de $20 \%$, uma vez que esse percentual é o mais adequado para se trabalhar em compósitos particulados.
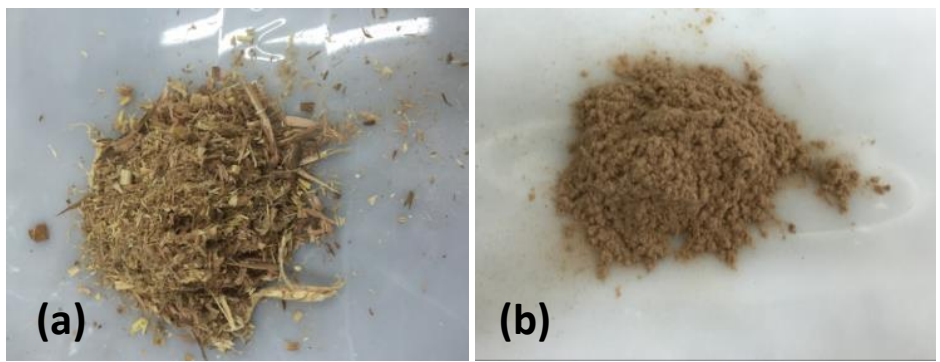

Figura 2: (a) Raspa da madeira e (b) Pó da madeira.

\subsection{Preparação Dos Compósitos}

Foram feitos três corpos de prova para cada configuração estudada, sendo eles poliuretano puro (PU/Puro), poliuretano com raspa de madeira (PU/Raspa) e poliuretano com pó de madeira (PU/Madeira). Para confecção dos corpos de prova, utilizou um misturador eletrônico, para 
facilitar a homogeneização dos componentes (matriz/reforço). Ao final da mistura dos componentes, a cura dos corpos de provas durou 24 horas, a temperatura ambiente $\left( \pm 25^{\circ} \mathrm{C}\right)$. $\mathrm{O}$ molde usado na confecção dos corpos de prova tinha formato cúbico retangular feito de aço inoxidável com volume final de $1000 \mathrm{~cm}^{3}$ (Fig. 3-a). Por fim, os corpos de prova foram desmoldados. Utilizou-se também uma prensa para reter o excesso da expansão dos corpos de prova, visto que o poliuretano tem essa característica. Obtendo-se como resultado as amostras, como mostrado na Fig. 3-b.
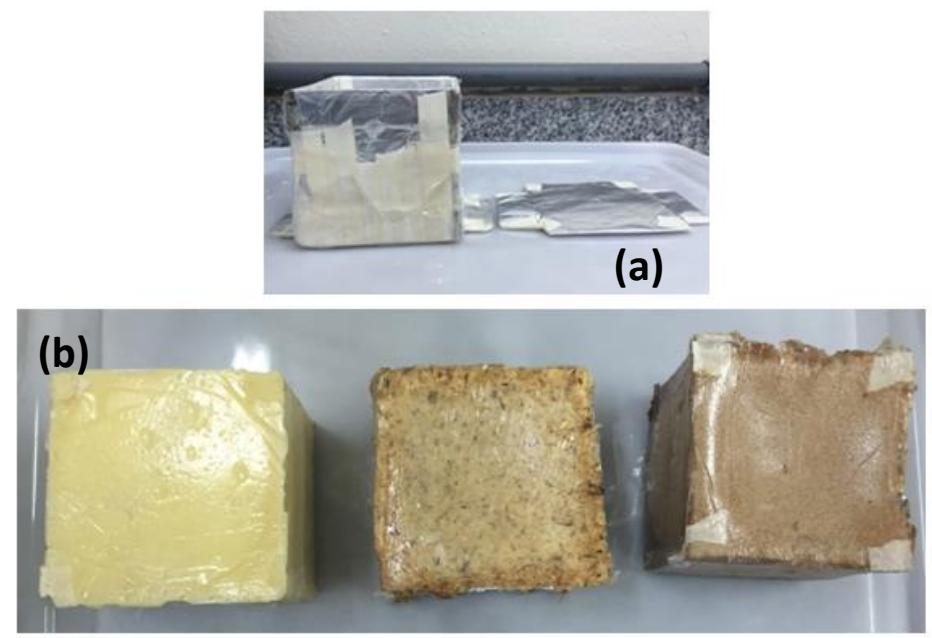

Figura 3 : (a) Molde metálico; (b) Corpos de Prova: PU/Puro; PU/Pó; PU/Raspa.

\subsection{Ensaio de Densidade Aparente}

O referido ensaio teve como principal objetivo determinar a densidade aparente do ecocompósito. O ensaio foi realizado nas três compósitos fabricados: PU/Puro, Pu/Pó e PU/Raspa, esses possuíam formato de cubo com uma média de lado de $2,5 \mathrm{~cm}$, como mostrado na Fig. 4 . A densidade dos compósitos foi determinada com a utilização de um densímetro da marca Gehaka e modelo DSL 910 . O ensaio foi realizado a uma temperatura ambiente $\left( \pm 25^{\circ} \mathrm{C}\right)$. A densidade é definida como sendo a massa por unidade de volume (FOX, 2001), podendo ser expressa pela Eq. 2:

$$
d=\frac{m}{v}
$$

Onde:

$d$ é a densidade $\left[\mathrm{g} / \mathrm{cm}^{3}\right]$;

$m$ é a massa [g];

$v$ é o volume $\left[\mathrm{cm}^{3}\right]$. 


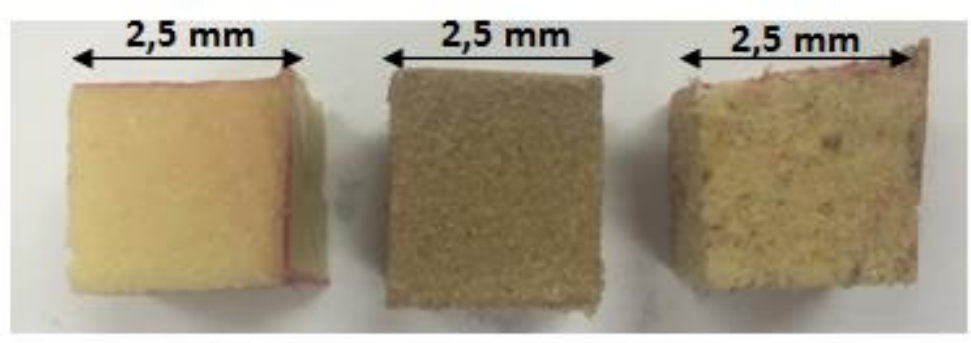

Figura 4: Corpos de prova para o ensaio de densidade

\subsection{Ensaios Térmicos}

O ensaio térmico utilizou-se o equipamento KD2 Pro (Fig. 5), esse possui um sensor SH-1 (agulhas térmicas duplas) que serve para capturar os valores, após 2 minutos de inserção nos corpos de prova. O ensaio ocorreu da seguinte forma: foram feitas seis medições em cada corpo de prova, onde se obteve a condutividade térmica, calor específico, resistividade térmica e difusividade térmica. Ou seja, realizamos um total de dezoito medições (seis em cada um dos três corpos). A penetração do sensor de medição teve o auxílio de uma furadeira para não danificar as hastes do mesmo. Esses furos estavam distantes entre si com uma média de $2 \mathrm{~cm}$.

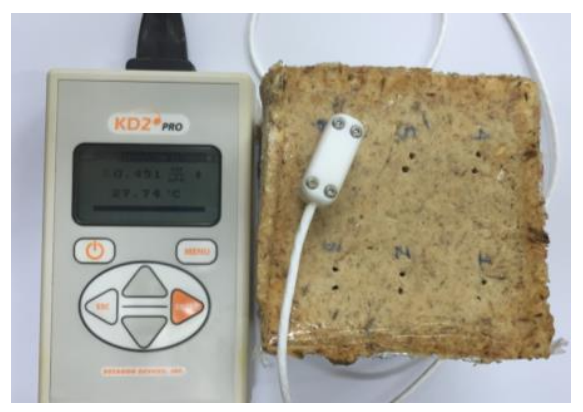

Figura 5: Equipamento em uso juntamente com um corpo de prova.

\section{RESULTADOS E DISCUSSÕES}

\subsection{Ensaio de Densidade Aparente}

Pela Equação 2, verificamos que para um volume constante, a massa e a densidade são diretamente proporcionais, o que se verifica em nosso experimento. Visto que ao retirarmos certa concentração de PU e substitui- lá por pó ou raspa a densidade diminuiu, já que para mesmas concentrações o PU é mais pesado que a raspa, a qual possui um peso bastante semelhante ao pó.

Tabela 1: Densidade dos compósitos.

\begin{tabular}{c|c}
\hline Configurações & Densidade $\left(\mathbf{g} / \mathrm{cm}^{\mathbf{3}}\right)$ \\
\hline PU Puro & 0,352 \\
PU mais Raspa & 0,275 \\
PU mais Pó & 0,270 \\
\hline
\end{tabular}




\subsection{Ensaios Térmicos}

A partir das seis medições para obtenção das propriedades térmicas (condutividade térmica, calor específico e difusividade térmica), em todos os corpos de prova, obtivemos uma média das suas propriedades térmicas (Tab. 2).

Tabela 2: Média das medições.

\begin{tabular}{c|c|c|c}
\hline Propriedades & PU/Puro & PU/Pó & PU/Raspa \\
\hline $\mathrm{k}[\mathrm{W} / \mathrm{mK}]$ & 0,0840 & 0,0495 & 0,0493 \\
$\mathrm{Cp}\left[\mathrm{J} / \mathrm{m}^{3} \mathrm{~K}\right]$ & 894 & 477,1667 & 446,8333 \\
$\alpha\left[\mathrm{mm}^{2} / \mathrm{s}\right]$ & 0,0950 & 0,1038 & 0,1096 \\
\hline
\end{tabular}

\subsubsection{Condutividade Térmica}

Como já sabemos, a condução é um processo de transferência de energia, ao longo do sistema, partícula a partícula. Uma partícula ao receber energia aumenta seu estado de vibração e dessa forma, acaba por transferir energia às partículas vizinhas. Assim, quanto mais partículas, mais rapidamente ocorre essa transferência, resultando em uma maior condutividade térmica. Isso foi verificado em nosso trabalho, visto que o PU/Puro, material dentre os três com maior densidade (massa por unidade de volume), obteve a maior condutividade. Enquanto que o PU/Pó e o PU/Raspa apresentaram valores de condutividade bem próximos, assim como seus valores de densidade. Em termos de percentual de retenção para essa propriedade térmica, o PU/Pó e o PU/Raspa, apresentaram 58,9 \% e 58,7\%, respectivamente, se comparado ao PU/Puro, valores estes quase que constantes, podemos dizer que houve uniformidade na medição e no material como um todo.

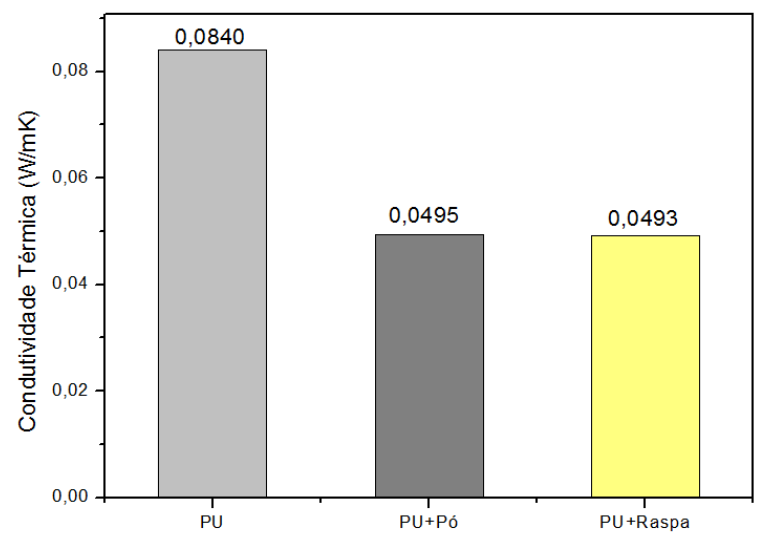

Figura 6: Condutividade Térmica.

\subsubsection{Calor Específico}

O calor específico mede a dificuldade de um corpo variar a sua energia. Um corpo com dificuldade de variar sua temperatura é um mal condutor, ou seja, é um bom isolante térmico, o que indica um baixo valor de condutividade térmica. Assim, calor específico e condutividade térmica são grandezas inversamente proporcionais. Porém, nem todos os materiais seguem essa regra, como por exemplo, o alumínio puro e sua liga 2024-t6, como mostra a Tab. 3. 
Tabela 3: Propriedades térmicas do alumínio.

\begin{tabular}{c|c|c}
\hline Propriedades & Alumínio Puro & Liga 2024-T6 \\
\hline Densidade $\left(\mathrm{kg} / \mathrm{m}^{3}\right)$ & 2702 & 2770 \\
Calor Específico $(\mathrm{J} / \mathrm{kgK})$ & 903 & 875 \\
Condutividade Térmica $(\mathrm{W} / \mathrm{mK})$ & 237 & 177 \\
\hline
\end{tabular}

A partir da Tabela 3, verificamos que para esses dois materiais, o calor específico, a densidade e a condutividade térmica são diretamente proporcionais, o que foge do padrão, mas pode ser explicado pelo fato de que quanto mais matéria, maior a possibilidade de armazenamento de calor. Na Figura 6 abaixo são mostrados os valores do calor específico para os corpos de prova estudados. Os valores para o calor específico dos compósitos que apresentaram o reforço da madeira caíram consideravelmente em relação a essa propriedade térmica, o PU/Pó caiu em torno de $53 \%$ chegando a um patamar de $477 \mathrm{~J} / \mathrm{m}^{3} \mathrm{~K}$, já o PU/Raspa obteve uma redução de calor específico em torno de $49 \%$, apresentando um valor de $446 \mathrm{~J} / \mathrm{m}^{3} \mathrm{~K}$.

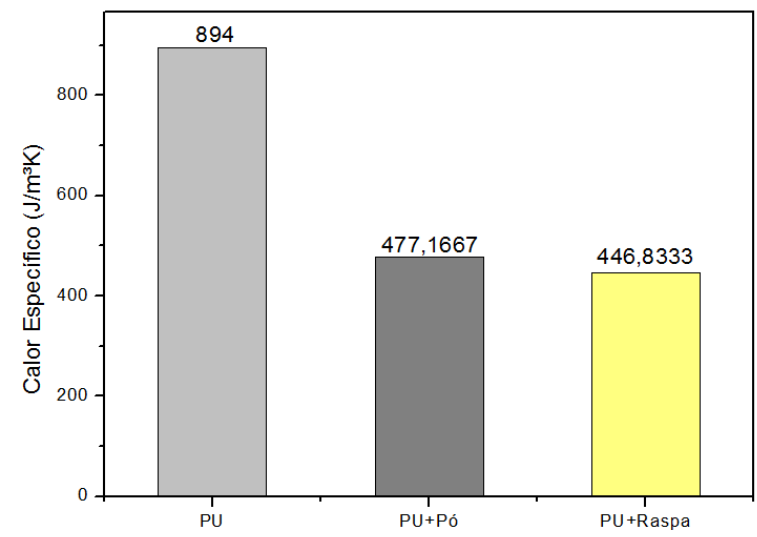

Figura 7: Calor Específco.

\subsubsection{Difusividade Térmica}

Conforme a Equação 1, tratada na seção 2.3, um aumento na condutividade térmica ocasiona um aumento da difusividade térmica, enquanto que um aumento na densidade volumétrica e no calor específico, gera uma redução na difusividade. Ou seja, condutividade é diretamente proporcional à difusividade, porém é inversamente proporcional a densidade e ao calor específico. Analisando os dados anteriores, percebemos um aumento nesses três parâmetros e concluímos que a difusividade diminuiu por termos das duas grandezas desfavoráveis, contra uma favorável a esse aumento. Isso pode ser visto na Fig. 8. Em valores numérico, a difusividade térmica apresentou um comportamento crescente para as diferentes configurações, como era de se esperar o PU/Puro, obteve a menor difusividade térmica, em torno de $0,095 \mathrm{~mm}^{2} / \mathrm{s}$, o ganho nessa propriedade térmica representou para as demais configurações um aumento de 9,26\% e 15,36\% para o PU/Pó e o PU/Raspa, respectivamente. 


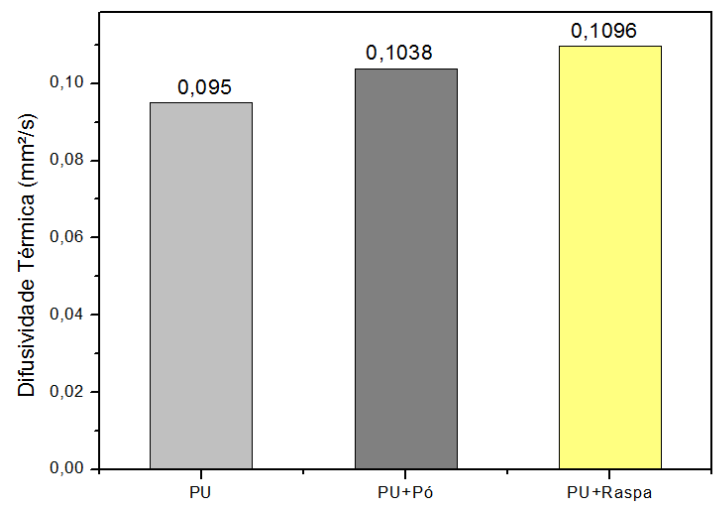

Figura 8: Difusividade Térmica.

\section{CONCLUSÃO}

Esse trabalho permitiu um estudo térmico de um eco compósito feito de rejeito de madeira com poliuretano a base de mamona, onde se tem como objetivo seu uso como material isolante.

Analisando-se suas propriedades térmicas e a suas densidades, verificamos algumas relações de proporcionalidade, como condutividade térmica, densidade aparente e calor específico apresentarem-se diretamente proporcionais;

Constatou-se também que os corpos de provas reforçados com madeira, seja pó ou raspa, apresentaram valores térmicos bem próximos;

Os compósitos reforçados com a madeira apresentaram menor condutividade térmica quando comparada com o PU puro, isso implica em uma melhor aplicação para materiais isolantes térmicos.

\section{REFERÊNCIAS BIBLIOGRÁFICAS}

1. CALLISTER JR.W.D, Fundamentos e Ciências e Engenharia de Materiais: Uma Introdução. Rio de Janeiro: LTC, 2002.

2. CORREA, C. A.; FONSECA, C. N. P.; NEVES, S. Compósitos Termoplásticos com Madeira. Polímeros: Ciência e Tecnologia, vol. 13, no 3, p. 154-165, 2003.

3. CUNHA, R. A. D. Influência do Envelhecimento Acelerado nas Propriedades Mecânicas do PRFV Quando Imerso em Petróleo. Trabalho de Conclusão de Curso. Universidade Federal do Rio Grande do Norte, 2015.

4. FOX, R. W.; MC DONALD, A. T. Introdução à Mecânica dos Fluidos. 5a Edição. Rio de Janeiro: LTC, 2001.

5. FREIRE JR, R. C. S. Fadiga de Alto Ciclo em Compósitos de PRFV. Propriedade Mecânica e Prevenção de Falha. 2005. Tese de Doutorado - Universidade Federal do Rio Grande do Norte, p.1-2.

6. GALVÃO, A. C. P. Obtenção e Caracterização de um Compósito de Poliuretano de Mamona e Pó de Vidro para Aplicações em Isolantes Térmicos. Dissertação de Mestrado. Universidade Federal do Rio Grande do Norte. 2014.

7. HERAKOVICH, C. T. Mechanics of Fibrous Composites.460 p, 1997. 
8. INCROPERA, F. P.; DEWITT, D. P.; BERGMAN, T. L.; LAVIANE, A. S. Fundamentos de Transferência de Calor e Massa. 6ạ Edição. Rio de Janeiro: LTC, 2008.

9. MATTHEWS, F. L.; RAWLINGS, R. D.; Composite Materials: Engineering and Science, Chapman \& Hall., 1994.

10. MENDONÇA, P.T.R. Materiais Compostos \& Estruturas-Sanduíches. Barueri: Manoele, 2005.

11. RAPIN, P. Manual do Frio. Editora Hemus, 8o edição, 2001, 462p.

12. SHACKELFORD, J. F. Ciência dos Materiais. 6ạ Edição. São Paulo: Pearson, 2008.

13. TORREIRA, R.P. Isolantes térmicos - frio \& calor. Fulton Editora Técnica LTDA. 1980,1024 p. 